\title{
Microplastic abundance in the atmosphere of Krakow (southern Poland)
}

\author{
K. JAROSZ ${ }^{*}$, R. JANUS ${ }^{2,3}$, M. WĄDRZYK ${ }^{2,3}$, \\ W. WILCZYNSKA-MICHALIK ${ }^{4}$, M. MICHALIK ${ }^{1}$, \\ ${ }^{1}$ Institute of Geological Sciences, Jagiellonian University, \\ Gronostajowa 3a, 30-387 Krakow, Poland \\ (*correspondence: kinga.borek@uj.edu.pl, \\ marek.michalik@uj.edu.pl) \\ ${ }^{2}$ AGH University of Science and Technology, Faculty of \\ Energy and Fuels, A. Mickiewicza 30, 30-059 Krakow, \\ Poland (rjanus@agh.edu.pl) (wadrzyk@agh.edu.pl) \\ ${ }^{3}$ AGH University of Science and Technology, AGH Centre of \\ Energy, Czarnowiejska 36, 30-054 Krakow, Poland \\ ${ }^{4}$ Institute of Geography, Pedagogical University of Cracow, \\ Podchorążych 2, 33-332 Krakow, Poland \\ (wanda.wilczynska-michalik@up.krakow.pl)
}

Microplastic (MP) abundance in the environment is being a subject of widely scoped research. So far the aquatic environments are being taken under consideration as the main medium of MP dispersion and its potential concern to human health. However, atmospheric fallout analysis confirms that air is also polluted with MP and human exposure to airborne MP could prove to be even more alarming.

The aim of the research was to recognise the MP abundance in urban area of Krakow, the city of 771 thousand residents that is also yearly visited by 20 times as many tourists. The research began in late 2018 and has proven the MP atmospheric pollution presence in Krakow.

Microplastic particles of different size $(0.1-5 \mathrm{~mm})$ and forms were observed. The majority of microplastic was recognized as secondary in terms of source, and fibrous in terms of form. A few kinds of the most popular synthetic polymers (most notably: PP, Nylon, PET, LDPE) were recognized using Py-GC/MS and ATR-FT-IR spectroscopy techniques. Moreover, the observation of the environment's impact on MP fibers was made. SEM-EDS results indicated continuous degradation of synthetic polymers, also different mineral phases being attached to the MP surface.

Taken into account the amount, variety of sizes, degradation levels and environmental interaction traces of the MP found in Krakow the environmental impact of its occurrence seems to be high.

Acknowledgements. The research was financed with the Diamond Grant DI2017 021647.

The research was partially carried out using the infrastructure of the AGH Centre of Energy, AGH University of Science and Technology. 